\title{
La perméabilité des sphères éducatives et de socialisation: I'exemple du bénévolat des jeunes ${ }^{1}$
}

\section{Saskia Weber Guisan et Sandrine Cortessis}

Cet article repose sur les résultats d'une recherche portant sur le développement de compétences chez les bénévoles de 16 à 25 ans. Le corpus de cette recherche qualitative comprend les transcriptions de 41 entretiens semi-directifs. Cette contribution propose de mobiliser les notions d'alternance, d'altérité, d'étonnement et de boundary crossing pour comprendre la place et le rôle que prend l'activité bénévole des jeunes parmi les autres sphères de leur existence. Nous analysons ici neuf témoignages tirés de ce corpus pour rendre compte de la manière dont ces jeunes négocient des passages, des ouvertures, ou au contraire érigent des frontières entre leur sphère de bénévolat, leur sphère de formation et leur sphère professionnelle. Les résultats montrent que sous certaines conditions, le bénévolat peut être considéré comme un lieu d'apprentissage informel offrant des ressources en termes de développement de compétences, de construction identitaire et de projection de soi.

\section{Introduction}

L'objet de cet article est de se pencher sur la place et le rôle que prend l'activité bénévole des jeunes parmi les autres sphères de leur existence. Pour comprendre ces aspects, nous proposons de mobiliser les notions d'alternance, d'altérité, de boundary crossing et d'étonnement, dans une approche principalement sociologique, en nous appuyant sur neuf témoignages issus d'une recherche qualitative plus large (41 entretiens) portant sur le développement de compétences chez les jeunes bénévoles.

Nous commencerons par un cadrage de la question, puis donnerons quelques informations sur la recherche dont sont extraits les témoignages sur lesquels nous nous appuyons. Ensuite, nous développerons notre réflexion sur la base des notions précitées en les présentant au niveau théorique et en les articulant avec les données empiriques.

Dans un contexte d'apprentissage tout au long de la vie, notre société appelle ses citoyens à exploiter des ressources développées lors de parcours de moins en 
moins linéaires. Ainsi, comme le relève Heinz (2000, cité par Cortéséro, 2010), "confrontés à des opportunités de vie multiples, privés de toute garantie sur l'avenir, les individus multiplieraient les expériences autoformatives dans des sphères d'interaction diversifiées, où ils renégocient en permanence le sens de

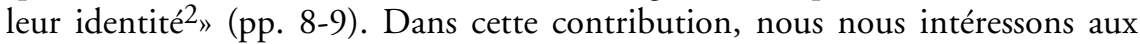
plus jeunes de ces individus aujourd'hui appelés à composer entre différentes sphères de socialisation, potentiellement éducatives, afin de construire leur futur.

Durant cette période bouillonnante et de plus en plus longue ${ }^{3}$ qu'est la transition entre l'adolescence et l'âge adulte, les insertions dans le monde familial, scolaire, professionnel et de loisir se superposent souvent (Becquet \& Cortéséro, 2015 , octobre). Les normes, savoirs et relations entretenues entre ces différentes sphères sont particulièrement susceptibles d'être interrogés, mis à l'épreuve et renégociés par les jeunes. Selon Galland (2011), on est passé d'un modèle de socialisation d'identification (basé sur la reproduction du modèle familial) à une socialisation d'expérimentation. Les parcours perdent alors en linéarité et sont souvent ponctués d'allers et retours. Bessin (2009, p. 16) parle à ce propos de parcours de vie de plus en plus "déstandardisés». Il y a une forme de brouillage des sphères qui étaient auparavant mieux délimitées et dont les frontières étaient plus marquées. Cette "porosité des sphères de la vie» (Bidart, 2006, p. 37 et p. 50) incite donc à une pluralité interprétative des différents rôles sociaux.

La notion de «sphère d'expérience» de Zittoun (2012) évoque cette pluralité, qui peut être à la fois interconnectée, distincte, investie ou désengagée:

Durant l'adolescence, la personne s'engage dans une pluralité de sphères d'expériences - de situations sociales qu'elle perçoit comme subjectivement distinctes. Dans une même journée, ou une même semaine, elle passe de l'école à son club de sport, de moments passés avec une bande de copains, à ceux en compagnie de sa famille ou une meilleure amie, ou encore seule à surfer sur internet ou à écouter de la musique. Ces sphères peuvent se multiplier, certaines peuvent être désinvesties; elles évoluent parfois en même temps, parfois à des rythmes différents. (pp. 14-15)

Parmi ces sphères d'expérience (familiale, amicale, scolaire, professionnelle, de loisirs ou de formation), nous nous penchons plus spécifiquement sur celle du bénévolat. Plutôt que de la considérer comme une sphère à part et imperméable, nous nous interrogeons sur ses relations et articulations dynamiques avec d'autres sphères. Pour illustrer notre propos, la sphère amicale peut prendre place à la fois dans la sphère professionnelle, la sphère de loisirs (sport par exemple) et la sphère associative (engagement caritatif par exemple).

Ce qui précède interroge aussi la question des sphères éducatives. Si la possibilité de développer des savoirs en dehors de l'école est admise depuis longtemps déjà (Dewey [1916/1938/2011] développe la notion de l'apprentissage par l'expérience; Lindeman [1925] évoque la question de l'apprentissage informel; Vygotski [1934/1997] s'intéresse au rapport entre concepts scientifiques et 
concepts quotidiens), l'étude sur les différents lieux d'apprentissage ou leurs interrelations n'est pas encore aboutie. Dans le cas qui nous intéresse, c'est le monde associatif et plus précisément le bénévolat des jeunes qui est considéré comme un lieu d'apprentissage informel permettant le développement de compétences. Mais comme l'indique Brucy (2007), les difficultés commencent dès lors qu'il s'agit de faire reconnaître ces acquis extrascolaires, sachant que les savoirs construits dans un contexte donné prennent davantage de valeur si l'on peut établir un lien entre eux et les autres contextes.

Cette partie introductive nous amène à formuler les questions suivantes:

- Comment les jeunes perçoivent-ils le rôle et la place qu'occupe leur activité bénévole au sein des autres sphères de leur existence?

- Comment les jeunes bénévoles perçoivent-ils le type de rapport que les différents milieux de socialisation et d'apprentissage dans lesquels ils évoluent entretiennent les uns par rapport aux autres?

- Les jeunes bénévoles négocient-ils des passages, des ouvertures ou au contraire érigent-ils des frontières entre la sphère bénévole, la sphère scolaire et la sphère professionnelle?

Nous y répondrons en prenant appui sur des données empiriques provenant d'une recherche que nous présentons succinctement puis nous mobiliserons les notions d'alternance, d'altérité, d'étonnement et de boundary crossing pour comprendre les résultats obtenus.

\section{Une recherche sur le bénévolat des jeunes}

La recherche dont sont issus les extraits rapportés dans cet article porte sur les apprentissages développés par des jeunes de 16 à 25 ans au cours de leur activité bénévole. Voici tout d'abord quelques chiffres sur le bénévolat en Suisse qui permettent de contextualiser le phénomène: en Suisse, l'activité bénévole occupe une place importante. D'après l'Office fédéral de la statistique et la Société suisse d'utilité publique (2015), pour la tranche d'âge 15-24 ans, 18,8 \% des femmes et $18,9 \%$ des hommes «se consacrent à au moins une activité non rémunérée dans le cadre d'organisations ou d'institutions ${ }^{4}$ (p. 4) pour environ $20 \%$ tout âge confondu. Selon la dernière enquête de l'Observatoire du bénévolat (Freitag, Manatschal, Ackermann \& Ackermann, 2016):

Les jeunes adultes, à l'instar des adultes plus âgés, s'engagent bénévolement pour des motifs altruistes ou de mise en valeur de soi-même. Toutefois, les 15-34 ans prennent plus en compte certains aspects liant l'engagement bénévole, la qualification, la formation continue et l'enrichissement personnel que les bénévoles de plus de 34 ans. (pp. 5-6)

L'objectif principal de notre recherche consiste à identifier les conditions qui favorisent le potentiel formateur de l'environnement bénévole (considéré comme 
un lieu d'apprentissage informel), notamment en termes de ressources disponibles. Nous avons mené 41 entretiens semi-directifs en ciblant un public de jeunes provenant d'associations affiliées au Conseil suisse des activités de jeunesse (CSAJ). C'est par l'intermédiaire du CSAJ que nous avons approché la plupart de bénévoles interviewés. Ces derniers évoluent donc principalement entre pairs dans des associations généralement réservées aux jeunes. Les différentes associations répertoriées par le CSAJ entrent dans les catégories d'activités suivantes: vie sociale et politique $(n=19)$, culturel et événementiel $(n=8)$ et encadrement de jeunes $(n=14)$. Nous avons veillé à interviewer 22 jeunes femmes et 19 jeunes hommes provenant d'associations de tailles variables, de plusieurs niveaux de structuration et poursuivant différents types de missions. Nous voulions examiner si la structure influence l'investissement et l'apprentissage chez les jeunes. Nous avons également cherché à interroger des bénévoles ayant des parcours scolaires variés (apprentissage, haute école spécialisée [HES], université). Cette variété de profils sociologiques était importante, ceci dans le but de ne pas restreindre l'analyse à un groupe particulier, ce qui n'était pas notre objectif.

En nous inspirant des travaux de Simonet (2010) sur les carrières bénévoles, nous avons invité les jeunes à revenir sur les différentes étapes de leur parcours bénévole en s'arrêtant principalement sur les trois moments suivants: l'entrée dans l'activité bénévole, l'évolution en milieu bénévole et la sortie éventuelle de l'activité bénévole. Outre cet axe temporel, nous avons porté une attention particulière à la manière dont les interviewés établissent des liens entre les sphères bénévole, scolaire, professionnelle et privée.

Adoptant une posture compréhensive (Kaufman, 1996), nous n'avions pas pour but de procéder à une reconstitution objective de la réalité, mais plutôt de permettre aux interviewés de revenir sur les principales étapes de leur parcours bénévole et de mettre en récit leur expérience subjective, afin de lui donner un sens et une forme.

Les entretiens, d'une durée moyenne d'une heure, ont fait l'objet d'une transcription verbatim. Toutefois, pour alléger le corpus et rendre la lecture plus aisée, nous avons supprimé quelques tics de langage et amélioré parfois la syntaxe. Les transcriptions ont été anonymisées au niveau des noms propres ainsi que des petites associations trop facilement repérables. Nous avons ensuite effectué une analyse de contenu, en adoptant une démarche principalement inductive, avec le logiciel $\mathrm{NVivo}^{\circledR 5}$ qui nous a permis de coder les thèmes récurrents repérés dans les entretiens.

Cet article ne restitue pas l'ensemble de notre recherche, mais s'appuie sur neuf entretiens qui témoignent d'une part de la manière dont les interviewés traversent successivement plusieurs sphères et se trouvent confrontés à différents statuts identitaires, mais aussi à différentes pratiques, et d'autre part des significations qu'ils attribuent à ces expériences. Nous nous attarderons plus longuement sur le témoignage d'une jeune femme, Tiziana, qui nous semble emblématique de notre propos. 


\section{Une alternance nourricière}

Pour comprendre ce qui se joue lors des passages entre ces différentes sphères de socialisation ou espaces éducatifs, la notion d'alternance nous semble offrir une clé de lecture intéressante. Nous adoptons ici une acception large de l'alternance, qui ne se réduit pas au dispositif consistant à alterner, pour une personne en formation, entre un lieu de formation et un lieu de production (en Suisse, on parle d'apprentissage dual tandis qu'en France, on parle de formation par ou en alternance). Dans cette acception large, nous retenons spécifiquement la racine latine alter qui signifie autre. Une première acception de l'alternance telle que nous l'entendons revient à alterner entre un espace et un autre, ce qui évoque donc l'idée d'expérimenter un autre espace, avec d'autres règles, interlocuteurs, objectifs et valeurs. Cohen-Scali (2000) parle même de «situation d'émigration» (p. 48) pour définir l'alternance, ce qui rappelle immanquablement l'idée d'un déplacement, qui peut être physique, psychique ou social.

Notre public cible nous a permis de rencontrer des personnes qui, parallèlement à leur activité bénévole, sont soit en formation, soit en transition entre l'école obligatoire et une formation ou entre une formation achevée et le début de leur vie professionnelle, soit encore en début d'activité professionnelle. Ainsi, leur activité bénévole implique une forme d'alternance entre leur vie scolaire, leur formation professionnelle, leurs études universitaires, leur recherche d'emploi ou de place d'apprentissage ou encore leur travail salarié.

Barbier (1997) définit l'alternance comme «un processus de construction de configurations variées de différents espaces de socialisation [et de formation]» (p. 60). Le terme de "construction» suggère qu'il ne s'agit pas de juxtaposer différents lieux, mais bien de construire et articuler plusieurs lieux, comme l'exprime Barbara, 24 ans, employée de commerce et active dans une Jeunesse campagnarde:

Au niveau théorique, c'est beaucoup, ben justement le professionnel qui m'aide pour l'activité de bénévolat que ce soit pour l'organisation, on a un agenda, la réalisation d'une séance, des choses comme ça. Par contre le bénévolat m'apporte beaucoup au niveau privé et professionnel. C'est de la confiance en soi, parce qu'en fait on doit souvent prendre la parole devant des centaines de personnes ou bien en assemblée, on doit défendre son avis, voilà on débat. Donc ça, ça m'aide beaucoup tant au niveau privé que professionnel.

Nous avons pu constater plus haut que Barbara relie les différentes activités qu'elle investit et en mesure le potentiel nourricier. À l'instar de Barbara, Loïc, 23 ans, étudiant HES, moniteur de gymnastique et actif dans une Jeunesse campagnarde, a également très bien compris comment son expérience bénévole pouvait lui servir pour construire son avenir professionnel. En effet, son activité bénévole lui permet de mettre en pratique des savoirs théoriques enseignés à 
l'école. En d'autres termes, son activité bénévole fait office de stage. Il construit ainsi son cheminement entre plusieurs expériences qui comprennent à la fois des formations formelles, et des expériences associatives. Ces résonnances entre deux espaces de pratiques permettent de consolider des compétences et donnent une cohérence forte à son parcours.

Je me suis mis dans la commission média, pub, marketing parce que c'est exactement ce que moi je suis en train de faire dans ma formation donc gestion de la page Facebook et ce genre de truc... ça me permet de mettre en pratique ce que j'apprends au cours.

L'expérience bénévole de Loïc est au bénéfice de ses études et vice-versa, elle se situe dans cette interface entre formation et production, qui est une définition classique de l'alternance. Rappelons à ce sujet les deux dimensions de l'activité humaine (Vygotski, 1934/1997; Samurçay \& Rabardel, 2004) ${ }^{6}$ : (i) la dimension productive: en agissant, le sujet transforme le réel (réel matériel, social et symbolique) et (ii) la dimension constructive: en transformant le réel, le sujet se transforme lui-même. Envisager l'expérience bénévole sous ces deux dimensions permet d'en mesurer le potentiel à la fois formateur (dimension constructive) et producteur (en tant que transformation du réel).

\section{Devenir autre}

Si pour certains jeunes, l'activité bénévole introduit une forme de continuité avec leur parcours de formation ou leur parcours professionnel, d'autres en revanche marquent les frontières entre ces différentes sphères. Cette différenciation volontaire renvoie à une autre dimension de l'alternance qui est l'altérité. L'altérité évoque principalement la différence, mais est également intimement liée à la question de l'identité. À ce titre, les travaux de Ricœur (1990) montrent cette subtile articulation et indissociabilité entre identité et altérité. Dans son ouvrage «Soi-même comme un autre», Ricœur précise: "Au "comme", nous voudrions attacher la signification forte, non pas seulement d'une comparaison - soi-même semblable à un autre -, mais bien d'une implication: soi-même en tant que... autre» (p. 14).

\section{Les identités multiples}

Dans «L'homme pluriel», Lahire (2011) postule que l'individu traverse une multitude de sphères qui ne sont pas homogènes. Au lieu de considérer l'individu par rapport à un rôle spécifique (parent, professionnel, retraité, etc.), il propose une vision complexe de l'individu: un acteur pluriel. En effet, en reprenant le principe d'Archimède, «tout corps (individuel) plongé dans une pluralité de mondes sociaux est soumis à des principes de socialisation hétérogènes et parfois même contradictoires qu'il incorpore» (p. 50). Les individus ne sont donc pas 
forcément cohérents, ils ont différentes identités selon les espaces sociaux qu'ils fréquentent. Notre jeune public, dont la transformation identitaire est une caractéristique de cette période de transition entre l'adolescence et l'âge adulte, peut chercher à être reconnu dans certaines de ses identités et en rejeter d'autres.

Identité et altérité se répondent sans cesse dans des phases successives d'opposition/appropriation (Colin, 2001). Ainsi, Emma, 16 ans, lycéenne, bénévole dans une Association de prévention santé, s'oppose au monde scolaire où elle se sent considérée comme une enfant, alors qu'elle s'identifie au monde associatif qu'elle côtoie, dont elle s'approprie les codes et qui lui donne une place qui a du sens pour elle:

C'est vrai que j'ai l'impression qu'on nous traite comme des bébés à l'école. [...] Souvent j'ai l'impression qu'à l'école on apprend beaucoup de choses inutiles tandis que dans le travail que je fais dans l'association, je suis plus ou moins convaincue que dans une certaine mesure c'est utile ce que l'on fait et puis cela m'intéresse.

Ce discours sur le monde scolaire se retrouve aussi chez Fred, 20 ans, apprenti et bénévole dans une association d'entraide pour homosexuels, qui évoque le côté passif et ennuyeux de ses cours:

Au cours, j'ai envie de m'ouvrir les veines, tu es assis toute la journée, tu fais rien, tu gobes des infos, tu dois même demander l'autorisation pour aller aux toilettes.

Il exprime donc d'un côté, ce sentiment d'être traité de manière infantilisante, de l'autre celui d'être actif dans des rôles valorisants. Ainsi, certains jeunes peuvent, grâce au bénévolat, actualiser une facette valorisante de leur identité qu'on leur refuse ailleurs. C'est particulièrement le cas de Dario, 22 ans, étudiant HES et sapeur-pompier volontaire, qui relève lui aussi le caractère actif de son engagement bénévole et exprime sa volonté explicite de ne pas faire de liens entre ses études et sa sphère bénévole:

Moi je vous ai dit que je venais par passion, et c'est pour ça que je viens, c'est parce que ça me permet de me consacrer à quelque chose de complètement différent de ce que je fais la journée. Donc la journée je suis comme tout le monde, un étudiant, je prends mon ordinateur, j'écoute les cours, et puis le soir quand on vient faire les exercices, ben je change complètement de visage. Je viens, j'enfile ma tenue, mon casque et puis ben je pense qu'à ça. [...] Comparé à mes cours où on fait beaucoup de théorie, c'est du management, c'est de l'économie, c'est des principes, puis quand j'arrive aux pompiers c'est pas des principes, c'est le terrain, et puis ben il faut prendre rapidement des initiatives [...]. Alors, des liens entre mes études et les pompiers y en a pas tellement, et c'est justement ça qui me plaît.

Dario évoque une véritable transformation de lui-même le soir venu, au point qu'on pourrait même parler d'alter ego (l'autre moi). Ce côté extrêmement 
valorisant et héroïque de soldat du feu agit comme une transition métaphorique et comme un nouveau repère identificatoire extrêmement puissant: il s'agit de l'autre soi qu'il voudrait être, ou le soi en tant que pompier. On retrouve ici l'implication en tant qu'autre (et non la simple comparaison) mentionnée par Ricœur (1990) plus haut. Le statut obtenu lors de son engagement volontaire est par ailleurs tellement gratifiant qu'il aimerait en faire son métier. Il s'investit alors de manière assidue chez les pompiers volontaires, multipliant les cours de formation continue afin d'avoir ses chances de réussir le concours très exigeant pour devenir pompier professionnel. Dans son cas, son activité bénévole est une forme de test vocationnel et de préprofessionnalisation.

Pour Nicolas, 19 ans, maçon et membre d'une Commission cantonale de jeunes, l'expérience bénévole est un moyen d'expérimenter et d'apprendre ce que l'école ne lui a pas permis de mettre en œuvre, en raison de la filière dans laquelle il avait été orienté:

I.: Qu'as-tu l'impression d'avoir appris là?

Nicolas: ben l'organisation de séances, tout ce qui est rédaction d'ordre du jour, communiqué de presse, analyser le règlement, après analyses de textes parce qu'à l'école j'étais en $\mathrm{VSO}^{7}$, donc je n'ai jamais fait d'analyse de texte vraiment. Après j'ai été sélectionné pour partir en Côte d'Ivoire à l'assemblée parlementaire francophone pour représenter la Confédération où là le critère de sélection, c'était de rédiger, une rédaction sur quatre thèmes que l'on avait à choix et puis donc là, cela m’a poussé à écrire et à me triturer les méninges.

Cet «alter $[. .$.$] devient une occasion de développement cognitif et identitaire»$ (Petit, 2007, p. 96) particulièrement important pour Nicolas qui est confronté à de l'inédit et a surtout eu l'opportunité de s'en saisir. Ce dernier a également l'occasion de faire entendre sa voix et obtient une reconnaissance de ce qu'il peut apporter au monde, ce qui n'a pas été possible dans le cadre scolaire, ni actuellement dans sa profession:

I.: Qu'est-ce qui te plaît qui fait que tu es dans cette commission?

Nicolas: Je dirais le fait de pouvoir travailler sur des textes de loi, d'être écouté, de donner son opinion.

De manière générale, nous constatons que les bénévoles interviewés parlent positivement de cette expérience d'altérité, qu'ils la relient ou non à leur formation ou leur vie professionnelle. Cette expérience leur permet de devenir autre, d'obtenir un statut qui va de pair avec une forme de reconnaissance nécessaire au développement identitaire, mais aussi professionnel. La question identitaire est par ailleurs reprise par Kaddouri (2008), pour qui l'alternance est un espace de transition non seulement professionnel, mais aussi identitaire, un espace de socialisation et un espace de construction des identités professionnelles (p. 173). 
Franchir les frontières pour se développer

Les travaux autour de la notion de boundary crossing sont également pertinents pour comprendre les passages entre différents contextes et ce qui s'y joue, notamment en termes d'apprentissages et de construction identitaire. Ce «franchissement de frontières» est compris par Suchman (1994) comme «entrer dans un territoire avec lequel on n'est pas familier et jusqu'à un certain point,

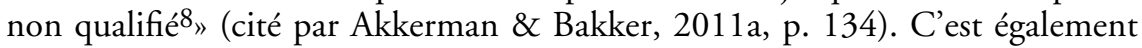
ce qu'évoque Zittoun (2012): «Dans toute situation perçue comme nouvelle, la personne doit définir de nouvelles manières d'agir et de comprendre; chaque sphère d'expérience demande donc des processus d'apprentissage, d'acquisition de connaissances et de compétences ${ }^{\prime \prime}$ (p. 15).

L'intérêt de ce concept est qu'il va au-delà de la simple notion de transfert. En effet, le transfert est unidirectionnel, tandis que le boundary crossing inclut des allers et retours, des alimentations mutuelles et des interactions entre les pratiques. Il enrichit donc la notion de transfert, notamment en établissant des ponts, des relations et des interactions entre des contextes culturellement et socialement différents (Akkerman \& Bakker, 2011b). Ainsi, l'idée d'enrichissement mutuel se superpose à la dichotomie entre différents contextes.

Pour illustrer cette notion, nous nous appuyons plus spécifiquement sur le témoignage de Tiziana, qui catégorise de manière très nette les différentes sphères qu'elle côtoie, notamment avec la dichotomie entre «monde jeune» et «monde adulte». Issue d'un milieu populaire, de parents immigrés, Tiziana, 20 ans, a eu une scolarité laborieuse et son apprentissage d'employée de commerce est également source de difficultés. Alors que selon ses dires elle ne connaît rien à la politique, elle intègre une Commission cantonale de jeunes, sous l'égide du Parlement des jeunes ${ }^{10}$ et en devient même la présidente pendant un an.

Ainsi, Tiziana traverse plusieurs sphères d'expérience (pour reprendre la notion de Zittoun, 2012): les cours à l'école professionnelle, les différents lieux de son apprentissage dual, son club de foot, sa famille, son quartier populaire et finalement la Commission cantonale des jeunes. Elle s'adapte plus ou moins bien à ces différentes sphères, mais se heurte à une difficulté majeure: celle de l'interaction avec ce qu'elle considère comme le monde adulte. Une tension s'installe entre le monde des jeunes qui "galèrent» auquel elle s'identifie et le monde adulte, sérieux, professionnel et hiérarchisé qu'elle doit intégrer petit à petit. À ce propos, les travaux de Galland (2011) montrent que pour certains jeunes, le statut d'adulte et professionnel n'est pas enviable, car il peut signifier une fermeture des possibles.

L'individu qui traverse successivement ou parallèlement plusieurs sphères d'expérience peut être confronté à différents statuts identitaires, ce qui engendre ainsi une position identitaire ambiguë. Cette forme de tension peut être vécue difficilement, mais peut également agir comme un levier permettant de briser les frontières (Akkerman \& Bakker, 2011b). Ainsi, Tiziana, relativement peu à l'aise dans son apprentissage en raison notamment de problèmes relationnels avec ses 
référents et des difficultés d'ordre scolaire, veut absolument réussir son Certificat fédéral de capacité (CFC) aux yeux de son autre sphère - celle de la Commission des jeunes. Dit autrement, le fait d'appartenir à la sphère de la Commission des jeunes et de s'y sentir intégrée résout ou atténue en quelque sorte une difficulté éprouvée dans une autre sphère, celle de son contexte professionnel (c'est-à-dire apprentissage en entreprise).

Cela m’a donné aussi envie de réussir mon CFC parce que je ne voulais pas arriver à la dernière séance dépitée, et pas gérer ma séance devant les nouveaux membres, et puis devant la conseillère d'État, devant le nouveau chef du Service de la jeunesse. Pour moi, c'était quelque chose qui était juste! Et puis Alain croyait beaucoup en moi!

La manière dont Tiziana gère les différentes sphères dans lesquelles elle est impliquée est très parlante en termes d'identification et d'intégration. Lorsqu'elle débute à la Commission des jeunes, Tiziana annonce d'emblée:

La première chose que j'ai dite, c'est que moi j'y connais rien en politique: «Moi, je peux rien vous ramener au niveau politique, je peux juste vous ramener l'expérience des jeunes, parce que moi j'ai plein de groupes d'amis. J'ai connu des amis qui sont très riches. J'ai connu des amis qui sont très pauvres, qui sont dans la rue, qui ont des bagarres, qui sont rejetés par la société à cause de leur nationalité. Moi, je peux vous amener ça! Je peux vous amener la vie de mes amis! La vie des vrais jeunes qui sont dans la rue et qui connaissent ces problèmes de société de tous les jours!»

Pour s'intégrer dans la Commission des jeunes, Tiziana d'une part apporte son expérience de «vraie jeune», d'autre part adopte certains codes qui lui semblent importants dans ce nouveau contexte. Bien qu'il s'agisse d'une activité bénévole, elle parle d'une attitude professionnelle à intégrer, notamment au niveau de son habillement ou encore par rapport à son rôle, parfois directif, de présidente:

Au niveau des séances, c'est vrai qu'il fallait quand même prendre un côté professionnel [...] C'est pas la fête au village quoi! [...] Exemple bête, je venais toujours en costard cravate, toujours, toujours, toujours parce que pour moi, c'était important!

Tiziana revêt donc le costume professionnel et sérieux de l'adulte, au propre comme au figuré. Il faut relever aussi que le costard cravate est une tenue masculine. On peut imaginer que pour Tiziana, le monde professionnel et adulte est plutôt un monde d'hommes, ce qui suggère qu'il y a pour elle également une frontière de genre à franchir.

Nous établissons un parallèle avec ce qui précède et la notion de boundary crossing events (ci-après $\mathrm{BCE}$ ) développée dans les travaux de Grossen, Zittoun et Ros (2014, p. 15 et pp. 19-20) dans le cadre d'une recherche portant sur les liens entre sphères d'expérience chez des élèves du secondaire II. Les BCE sont des éléments culturels (comme un événement social ou une expérience personnelle) amenés en classe par un élève ou un enseignant et en lien avec le cours 
suivi. L'inverse est également possible: un élément d'un cours peut être connecté à un fait (ou une actualité) qui se produit à l'extérieur du cadre scolaire. Le postulat de Grossen, Zittoun et Ros est que la connexion établie à l'aide des $\mathrm{BCE}$ permet de mieux gérer les tensions qui peuvent se produire entre deux sphères d'expérience. En transposant ce concept à la situation de Tiziana, nous pouvons avancer que le fait d'amener son expérience de «jeune qui connaît des difficultés» à la Commission des jeunes est une forme de BCE. En effet, elle apporte une contribution personnelle extrêmement connectée aux objectifs du Parlement des jeunes, qui se veut une instance représentative des jeunes. De l'autre côté, le fait que Tiziana s'habille de manière professionnelle pour les séances de la Commission peut être interprété comme une forme de BCE entre son lieu d'apprentissage (qui correspond au monde professionnel et adulte) et son engagement bénévole.

Ci-dessous, elle indique le changement de rôle qu'elle doit opérer: assumer son rôle de présidente, puis revenir à un statut de membre. On voit donc qu'à l'intérieur d'une sphère, différents statuts peuvent aussi créer des frontières qui doivent être franchies.

J'avais beaucoup ce côté professionnel et $\mathrm{j}$ 'arrivais à tenir ces membres que des fois sur des débats, cela devenait totalement farfelu! [...] Il faut essayer de gérer le timing, des choses comme ça! Donc, c'était beaucoup professionnel pour moi, à ce moment-là! Mais par la suite, donc à la fin des séances ou par exemple à la pause, je prenais totalement mon caractère normal, et puis je rigolais avec tout le monde! C'est vrai que moi, je ne me suis jamais prise la tête, par rapport au fait que j'étais présidente! [...] Juste au niveau des séances, j'étais juste légèrement plus haute, mais juste parce que je tenais la séance, c'est tout!

Comme nous l'avons déjà vu, le concept de boundary crossing permet d'aller plus loin que la notion linéaire de transfert et suscite des enrichissements mutuels. Dans la situation de Tiziana, son implication à la Commission des jeunes a contribué à sa réussite dans une autre sphère, en l'occurrence sa sphère de formation. Elle a en effet développé des compétences au sein de la Commission qu' elle a pu mobiliser dans le cadre de sa formation ou à un niveau plus personnel (comme se sentir plus à l'aise dans ses rapports avec les adultes):

Tout ce que j'ai acquis ici, au niveau professionnel, enfin extra-professionnel, au niveau de la commission, j'ai pu le mettre en œuvre dans l'apprentissage! Donc par exemple, l'écriture de mails, c'est vrai que j'étais plus à l'aise, je connaissais plus les formes de politesse. Comment gérer un mail, comment le faire par rapport aux personnes, par rapport au degré d'importance de la personne, donc ça ça m'a beaucoup aidée!

Cela m'a fait prendre de l'importance. J'ai pris beaucoup conscience des choses, enfin on voit la vie différemment et c'est vrai que cela m'a beaucoup aidée! Et oui, c'est vrai que j'ai beaucoup changé [...] quand on est jeune, on 
ne sait pas beaucoup comment se comporter face à un adulte [...] on ne sait pas forcément comment parler. [...] et cela m'a beaucoup aidée à être plus à l'aise avec des personnes adultes.

\section{Se confronter à d'autres sphères et s'étonner}

Tiziana évoque à plusieurs reprises son étonnement lorsqu'elle parle de son expérience à la Commission des jeunes. L'étonnement est provoqué par un «écart entre l'attendu et le réel» (Jobert \& Thievenaz, 2014, p. 38). Dans la même idée, s'étonner, selon Hameline (2014), "c'est comparer ce qui advient avec ce qui est, avec ce que l'on attendait, avec ce que l'on estimait devoir être. Et, de cette comparaison, éprouver du trouble $[. .$.$] , ou, tout au contraire [\ldots]$ espoir, ouverture émerveillement» (p. 11). Dans le cas de Tiziana, c'est un étonnement positif qui atténue probablement les préconceptions fortes qu'elle avait à propos du monde adulte et professionnel. S'étonner revient à déconstruire et à repenser ses catégories et de fait, à ouvrir ou déplacer ses propres frontières:

Ce qui m'a étonnée, c'est aussi la relation que j'ai eue avec Alain! Pour moi, c'était quelqu'un, ben heu voilà! Il me faisait un peu peur, et puis c'est quelqu'un de haut placé, mais cela s'est très bien passé! Et surtout le soutien que j'ai eu autour de moi! [...] En fait, tout m’a étonnée, parce que c'était vraiment une expérience où on se lançait et puis si ça marchait, ça marchait et si ça marchait pas, ça marchait pas! Et tout m’a étonnée en fait! Tout était super! Et Alain, nous le faisait comprendre par son étonnement à lui aussi, parce qu'il n'aurait jamais cru qu'on soit parti si loin en deux ans, surtout la première commission, donc oui tout m'a étonnée!

En retour, l'étonnement positif du coordinateur de la Commission cantonale des jeunes, Alain, renforce ce passage à double sens entre les sphères: ce n'est en effet pas uniquement Tiziana qui intègre et découvre un monde qu'elle ne connaît pas (c.-à-d. la Commission cantonale des jeunes), c'est aussi Alain, un adulte, qui entre dans le monde et les préoccupations de quelques jeunes du canton.

La frontière Jeunes/Adultes construite par Tiziana correspond à un contexte particulier ainsi qu'à une période spécifique de sa vie. Cependant, malgré cette dichotomisation forte Jeunes/Adultes, l'analyse montre comment Tiziana bouge dans ses représentations et comment certaines frontières deviennent perméables. Cette perméabilité s'opère en interaction avec d'autres jeunes, mais aussi avec des adultes, dont certains sont devenus des figures de référence. Alain incarne un modèle d'adulte valorisé par Tiziana. Elle s'est autorisée à le considérer comme un allié, et non comme un supérieur inaccessible, grâce à son implication à la Commission des jeunes. Il a probablement contribué à atténuer certaines frontières construites par Tiziana entre le monde jeune et le monde adulte, malgré la distance socioculturelle qui les sépare. D'après Tiziana, c'est aussi en partie grâce à Alain qu'elle a pu réussir son apprentissage, notamment par son soutien, mais aussi par le fait qu'il lui renvoyait une image valorisante qu'elle 
voulait préserver. Les travaux de Castets-Fontaine (2011) sur le cercle vertueux de la réussite scolaire montrent comment un individu pris dans un «engrenage de réussite» doit constamment entretenir ce succès, d'abord pour sa propre image, mais aussi afin de ne pas décevoir les personnes qui ont, d'après lui, permis cette réussite. Tiziana traverse donc ces frontières et redéfinit de nouvelles catégories: le monde adulte peut considérer le monde jeune avec bienveillance et respect, et les jeunes ont quelque chose à amener au monde adulte et réciproquement.

\section{Privilégier l'investissement dans la sphère bénévole, entre choix et contraintes}

Dans ce qui précède, nous avons suggéré que les jeunes interrogés placent leurs activités bénévoles au sommet de leur hiérarchie personnelle. Cette perception est-elle partagée par les adultes qui les entourent? En analysant, le rôle éducatif joué par les différents acteurs que sont, entre autres, la famille, l'école, les associations ou encore les pairs, Becquet et Cortéséro (2015, octobre) observent des ruptures et incompatibilités entre les différents acteurs éducatifs, tant du point de vue des objectifs poursuivis, que des manières de faire et des contenus. Selon Dubet (2014), l'idée suivante serait cependant largement partagée par l'ensemble des acteurs éducatifs adultes: «L'école fonctionne aujourd'hui comme une gare de triage dans laquelle les jeunes jouent pour l'essentiel leur avenir social puisque les diplômes décident de la position professionnelle que les individus occuperont» (p. 26). Dans cette logique, et toujours selon Dubet, les jeunes encore sous la tutelle de leurs parents jouent leur autonomie et donc leur latitude à investir des espaces sociaux extrascolaires, à l'aune de leurs résultats scolaires.

C'est ainsi que, selon Tiziana, son entourage a tendance à placer la scolarité comme étant l'activité la plus importante et à reléguer l'activité bénévole à un simple loisir:

Ma responsable ne croyait absolument pas en moi, ma conseillère non plus! Elles pensaient que j'allais complètement louper mon CFC, parce que justement, j'avais le bénévolat, j'avais ensuite le football, j'avais ensuite les examens! Pour elles, cela faisait beaucoup! Elles pensaient que je n'étais pas assez forte mentalement pour réussir à concilier les trois en fait. Et clairement, ma responsable m'a dit d'arrêter! Elle m'a dit que je devais totalement arrêter la commission, que je devais mettre ma priorité sur mon apprentissage.

Nous retrouvons ici le cas de figure décrit par Dubet (2014): tant que l'élève réussit, son entourage éducatif se soucie peu de la manière dont il gère ses différentes activités. En revanche, dès lors qu'une élève comme Tiziana rencontre des difficultés scolaires, les adultes interviennent de manière très directe pour rétablir le temps scolaire comme une priorité. Comme déjà évoqué, c'est pourtant grâce à son activité bénévole que Tiziana a eu l'opportunité d'accéder au statut 
valorisant de présidente de la Commission des jeunes. À cette occasion, elle a été interviewée par la télévision en présence de politiciens admiratifs. Elle nous confie que c'est cette reconnaissance obtenue sur la scène associative qui lui a donné la force de résister aux pressions scolaires et familiales et de concilier son activité bénévole et la réussite de ses examens.

Mener de front les activités bénévoles et les autres activités demeure cependant souvent un casse-tête, particulièrement pour les jeunes déjà engagés professionnellement comme Thomas, 19 ans, employé dans la construction et scout:

J'aimerais pas arrêter les scouts, mais y a un moment donné où, si je veux faire des activités, à côté en fait de mon boulot et à côté des scouts, je vais bien devoir à un moment donné trouver le temps à quelque part, donc ce qui fait que les scouts prennent de moins en moins de place dans mon temps, et ça c'est dommage, j'aurais voulu continuer plus, continuer, devenir chef, mais devenir chef c'est, c'est beaucoup de temps, ça prend beaucoup de temps.

Cet exemple donne une idée du lien entre l'investissement temporel dans l'activité bénévole et l'accès à des activités et à des statuts valorisants. Nous avons en effet constaté que celles et ceux qui ont le plus de temps à consacrer à leur activité bénévole sont également celles et ceux qui évoluent dans les milieux universitaires. Or, c'est dans ces milieux-là que le potentiel formateur de l'activité bénévole semble déjà le plus conscientisé et le plus valorisé, comme le mentionne Céline, 23 ans, étudiante et bénévole dans l'évènementiel:

En étant en Lettres, j’ai vraiment réalisé dès le départ que si je voulais avoir un job à la fin des études, il fallait que je fasse mille trucs à côté, que j’aie plein d'expériences.

\section{Perspectives: que peut offrir le bénévolat aux jeunes?}

Nos conclusions nous portent à considérer le bénévolat comme une sphère de développement potentiellement riche pour les jeunes entre 16 et 25 ans. En effet, il offre un espace d'apprentissage protégé, tout en étant proche du travail salarié par de nombreux aspects. Ainsi, les jeunes peuvent se confronter au monde (professionnel notamment), sans en subir des conséquences trop lourdes. Cet espace permet de vivre et de faire des expériences inédites, ouvre l'éventail des possibles et favorise la projection de soi et la construction identitaire dans un alter ego valorisant. La sphère bénévole est donc un espace «entre deux» qui n'est pas tout à fait du travail salarié, qui n'est pas pensé pour être une formation, et qui est plus qu'un simple loisir.

Nos résultats montrent que fréquemment, la sphère bénévole est envisagée par les jeunes comme une opportunité socialisatrice, réparatrice et formatrice. Les associations sont en effet perçues par les interviewés comme bienveillantes, 
leur offrant un statut valorisant permettant de repenser leur rapport à l'école et au monde professionnel. La possibilité de se confronter à l'altérité, à un autre monde, occasionne des transformations bénéfiques: avoir été un autre pendant un certain temps, c'est gagner de l'assurance et une expérience qui pourront être rejouées dans d'autres contextes. Le bénévolat ouvre ainsi des passerelles sociales que l'on retrouve relativement peu ailleurs dans l'espace social. Dans une certaine mesure, ces activités peuvent constituer une chance pour certains individus d'infléchir leurs parcours, notamment ceux issus de populations plus vulnérables, comme nous l'avons vu avec Tiziana. C'est grâce à la fréquentation de leur milieu associatif et à la mise entre parenthèses de leurs éventuels problèmes scolaires et familiaux que certains jeunes ont l'occasion de développer de nouvelles dispositions personnelles et de construire de nouveaux rapports à soi, au monde scolaire et au monde professionnel. Cela ne signifie pas pour autant que les bénévoles interviewés soient conscients de la valeur de leurs expériences extrascolaires. Chaque jeune situe en effet les savoirs développés dans son cercle associatif en opérant une hiérarchie sociale des savoirs. Cette construction s'effectue en interaction avec son entourage proche (famille, amis, enseignants, employeurs). Ainsi, nous observons qu'une même expérience réalisée dans le cadre bénévole pourra selon les cas être considérée comme une forme de préprofessionnalisation ou au contraire une simple bulle récréative n'ayant pour seul intérêt que d'offrir une évasion face à des impératifs scolaires ou professionnels ressentis parfois comme lourds. Ils opèrent cette intégration en se confrontant à leurs propres frontières et en y aménageant éventuellement des ouvertures. Que les différentes sphères soient liées ou non, les représentations qui s'y rattachent bougent, les frontières se déplacent et ouvrent donc des potentiels de développement. L'enjeu est alors pour ces jeunes «d'élaborer eux-mêmes le sens de leur intégration au sein des différentes sphères sociales» (Vermeersch, 2004, p. 683). Pour prolonger la réflexion, il serait intéressant de mener des entretiens à caractère biographique (Demazière \& Dubar, 1997) avec certains jeunes que nous avons interviewés afin de mieux comprendre, d'ici quelques années, ce qu'ils ont fait de leur expérience bénévole et comment celle-ci s'est intégrée sur l'ensemble de leur parcours.

S'il n'est pas question de formaliser ou d'instrumentaliser le bénévolat des jeunes, nous pensons qu'il est important de mieux le valoriser, en particulier auprès des acteurs éducatifs et des employeurs. Cette valorisation passe d'abord par une reconnaissance des compétences acquises par les bénévoles eux-mêmes. La prise de conscience s'effectue souvent par le récit oral ou écrit, en décrivant finement ses activités et ressources. C'est à ce titre que nous avons organisé des séances de portfolio (Aubret, 2000) avec un groupe de 15 jeunes bénévoles, dans le but de faire l'inventaire de manière structurée de leurs apprentissages et ressources pour qu'ils prennent conscience des compétences développées. Nous gageons que le fait de se reconnaître eux-mêmes participe d'une meilleure présentation et projection de soi, notamment pour formuler son CV, élaborer un dossier pour entrer dans une école ou se préparer pour un entretien d'embauche. 
L'enjeu à venir est donc de valoriser le potentiel du bénévolat des jeunes en termes de développement de compétences et de construction identitaire, tout en lui laissant son aspect librement consenti et informel qui en fait un lieu de formation alternatif, mais non obligatoire.

\section{Notes}

1 Nous tenons à remercier Michèle Grossen et les deux experts anonymes pour leurs critiques et suggestions constructives.

2 Nous nous appuyons sur la conception de l'identité de Dubar (1991) comme étant le résultat d'une double transaction: identité pour soi (axe biographique) et identité pour autrui (axe relationnel), ces deux axes étant négociés et renégociés tout au long de la vie.

3 "L'extension à la fois sociologique et statistique [INSEE] des frontières de la jeunesse conduit [...] à retenir 15 ans et 29 ans pour délimiter la jeunesse» (Roudet, 2012, p. 3).

4 Il s'agit de bénévolat organisé (ou formel) qui prend place dans des associations. Le bénévolat peut aussi être informel. Il s'agit alors d'aide à la famille ou aux proches et n'est pas soutenue par des structures. Dans le cadre de notre recherche, nous ne nous intéressons qu'au bénévolat organisé.

5 Logiciel d'analyse qualitative des données développé sur une approche grounded theory ou théorie ancrée (Glaser \& Strauss, 1967), qui postule que les chercheurs peuvent et doivent développer certaines théories en commençant par exploiter les données de terrain.

6 Ces deux dimensions ont déjà été émises par Karl Marx au 19e siècle.

$7 \mathrm{VSO}=$ voie secondaire à options. Dans le secondaire I du canton de Vaud, filière à exigences élémentaires préparant les élèves à l'entrée en formation professionnelle. Cette filière, fortement stigmatisée, a été supprimée depuis l'entrée en vigueur de la nouvelle Loi sur l'enseignement obligatoire (LEO) à la rentrée scolaire d'août 2013.

8 Traduit de l'anglais par les auteures.

9 Souligné par l'auteure.

10 La Fédération suisse des Parlements des Jeunes est une instance politiquement neutre qui offre un espace sur la scène politique pour la création de parlements par les jeunes et pour les jeunes.

\section{Références}

Akkerman, S. F. \& Bakker, A. (2011a). Boundary crossing and boundary objects. Review of educational research, 81, (2), 132-169.

Akkerman, S. F. \& Bakker, A. (2011b). Crossing boundaries between school and work during apprenticeships. Vocations and learning, 5, (2), 153-173.

Aubret, J. (2000). Le portfolio de ses acquis et compétences. Éducations, 18-19, 81-85.

Barbier, J.-M. (1997). Pour une approche ensemblière de l'alternance. Pour, 154, 57-64.

Becquet, V. \& Cortéséro, R. (2015, octobre). Socialisations juvéniles: des espaces éducatifs en interactions. Texte de cadrage du Colloque de l'Université de Cergy-Pontoise, Gennevilliers, France.

Bessin, M. (2009). Parcours de vie et temporalités biographiques : quelques éléments de problématique. Informations sociales, 156, (9), 12-21.

Bidart, C. (2006). Crises, décisions et temporalités: autour des bifurcations biographiques. Cahiers internationaux de sociologie, 120, (1), 29-57.

Brucy, G. (2007). Formation, certification: les métamorphoses de la reconnaissance. In F. Neyrat (Éd.), La validation des acquis d'expérience (pp. 15-39). Bellecombes-en-Bauges: Editions du Croquant.

Castets-Fontaine, B. (2011). Le cercle vertueux de la réussite scolaire. Le cas des élèves de Grandes Ecoles issus de «milieux populaires». Bruxelles: Éditions Modulaires Européennes.

Cohen-Scali, V. (2000). Alternance et identité. Paris: Presses Universitaires de France. 
Colin, P. (2001). Identité et altérité. Cahiers de Gestalt-thérapie, 1, 52-62.

Cortéséro, R. (2010). Au-delà du déclin de l'institution. Education et sociétés, 25, (1), 7-16.

Demazière, D. \& Dubar, C. (1997). Analyser les entretiens biographiques. Paris: Nathan.

Dewey, J. (2011). Démocratie et éducation suivi de Expérience et éducation. Paris: Armand Colin. (Originaux publiés en 1916 et 1938)

Dubar, C. (1991). La socialisation, construction des identités sociales et professionnelles. Paris: Armand Colin.

Dubet, F. (2014). Cultures juvéniles et régulation sociale. L'information psychiatrique, 1, (80), 21-27.

Freitag, M., Manatschal, A., Ackermann, K. \& Ackermann, M. (2016). Freiwilligen-Monitor Schweiz 2016. Zürich: Seismo.

Galland, O. (2011). Sociologie de la jeunesse. Paris: Armand Colin.

Glaser, B. \& Strauss, A. (1967). The discovery of grounded theory: Strategies for qualitative research. Chicago, IL: Aldine de Gruyter.

Grossen, M., Zittoun, T. \& Ros, J. (2012). Boundary crossing events and potential appropriation space in philosophy, literature and general knowledge. In E. Hjörne, G. van der Aalsvoort \& G. de Abreu (Éd.), Learning, social interaction and diversity - exploring school practices (pp. 15-34). London: Sense.

Hameline, D. (2014). Petite métaphysique de l'étonnement. Education permanente, 200, 9-15.

Jobert, G. \& Thievenaz, J. (2014). L'homo demirans ou l'homme qui s'étonnant, devient connaissant. Education permanente, 200, 33-42.

Kaddouri, M. (2008). Les formations en alternance entre transition, socialisation et constructions identitaires. In M. Kaddouri, C. Lespessailles, M. Maillebouis \& M. Vasconcellos (Éd.), La question identitaire dans le travail et la formation (pp. 173-185). Paris: L'Harmattan.

Kaufmann, J.-C. (1996). L'entretien compréhensif. Paris: Nathan.

Lahire, B. (2011). L'homme pluriel: les ressorts de l'action. Paris: Pluriel.

Lindeman, E. C. (1925). What is adult education? Manuscrit non publié, Columbia University, Butler Library Lindeman Archive, New York.

Office fédéral de la statistique \& Société suisse d'utilité publique (2015). Le bénévolat en Suisse 2013-2014. Travail et rémunération 03, 425-1500. Neuchâtel: OFS.

Petit, L. (2007). Fonction structurante de la discontinuité dans l'alternance. Education permanente, 172, 89-97.

Ricœur, P. (1990). Soi-même comme un autre. Paris: Seuil.

Roudet, B. (2012). Qu'est-ce que la jeunesse? Après-demain, 24, (4), 3-4.

Samurçay, R. \& Rabardel, P. (2004). Modèles pour l'analyse de l'activité et des compétences: propositions. In R. Samurçay \& P. Pastré (Éd.), Recherches en didactique professionnelle (pp. 163-180). Toulouse: Octarès.

Simonet, M. (2010). Le travail bénévole. Engagement citoyen ou travail gratuit? Paris: La Dispute.

Suchman, L. (1994). Working relations of technology production and use. Computer supported cooperative work, 2, 21-39.

Vermeersch, S. (2004). Entre individualisation et participation: l'engagement associatif bénévole. Revue française de sociologie, 4, (45), 681-710.

Vygotski, L. S. (1997). Pensée et langage (F. Sève, Trad.). Paris: La Dispute. (Original publié en 1934)

Zittoun, T. (2012). Usage de ressources symboliques à l'adolescence. Travaux neuchâtelois de linguistique, 57, 11-30.

Mots-clés: Bénévolat des jeunes, perméabilité des sphères, lieu d'apprentissage informel, boundary crossing, construction identitaire 


\section{Die Durchlässigkeit von Bildungs- und Sozialisations- bereichen: Das Beispiel der Freiwilligenarbeit Jugendlicher}

\section{Zusammenfassung}

Dieser Artikel basiert auf den Ergebnissen einer Studie zur Kompetenzentwicklung von Freiwilligen im Alter von 16 bis 25 Jahren. Die Datengrundlage dieser qualitativen Studie bilden die Transkripte von 41 halbstrukturierten Interviews. Um den Platz und die Rolle zu verstehen, die Freiwilligenarbeit von Jugendlichen innerhalb ihrer anderen Lebensbereiche einnimmt, schlägt dieser Beitrag vor, die Begriffe des Wechselspiels zwischen den Bereichen, der Andersartigkeit, des Staunens und des boundary crossing zu verwenden. Wir analysieren hier neun Interviews, um darzustellen wie diese Jugendlichen Verbindungen und Durchlässigkeit zwischen den Bereichen Freiwilligenarbeit, Ausbildung und Beruf herstellen, oder im Gegenteil Grenzen zwischen diesen Bereichen ziehen. Die Ergebnisse zeigen, dass Freiwilligenarbeit unter bestimmten Voraussetzungen als ein Ort des informellen Lernens verstanden werden kann, der Ressourcen in Bezug auf die Kompetenzentwicklung, die Identitätsentwicklung und die eigenen Zukunftsvorstellungen bereitstellt.

Schlagworte: Freiwilligenarbeit Jugendlicher, Durchlässigkeit von Bereichen, Ort informellen Lernens, boundary crossing, Identitätsentwicklung

\section{La permeabilità delle sfere educative e di socializzazione: l'esempio del volontariato giovanile}

\section{Riassunto}

Questo articolo si basa sui risultati di una ricerca sullo sviluppo delle competenze tra volontari di età compresa tra i 16 ei 25 anni. Il corpus di questa ricerca qualitativa include le trascrizioni di 41 interviste semi-strutturate. Questo contributo si propone di mobilitare le nozioni di alternanza, di alterità, di stupore e di boundary crossing per capire quale posto e ruolo assume l'attività di volontariato dei giovani, rispetto ad altre sfere della loro vita. Sono qui analizzate nuove testimonianze estratte dal corpus citato per mostrare come questi giovani negoziano dei passaggi e aperture, o al contrario erigono confini tra la loro sfera di volontariato, quella della formazione e quella professionale. I risultati mostrano che, in determinate condizioni, il volontariato può essere visto come un luogo d'apprendimento informale, che offre delle risorse in termini di sviluppo delle competenze, di costruzione identitaria e di progettualità personale.

Parole chiave: Volontariato giovanile, permeabilità, luoghi di apprendimento informale, boundary crossing, costruzione identitaria 


\section{The permeability of educational and socialization spheres: The example of youth volunteering}

\section{Abstract}

This article is based on the results of a research on competence development among 16 to 25 years old volunteers. The corpus of this qualitative research includes the transcriptions of 41 semi-structured interviews. This contribution proposes to use the notions of alternating between spheres, otherness, astonishment and boundary crossing in order to understand the place and the role that volunteering takes within the other spheres of young people lives. We analyse nine interviews taken from this corpus to understand the way in which these young people establish connections and permeability between their voluntary work sphere, their educational sphere and their professional sphere, or rather erect barriers between these spheres. Results show that under certain conditions, voluntary work can be considered as an informal learning situation offering resources in terms of competence development, identity construction and self-projection.

Keywords: Youth voluntary work, permeability of spheres, informal learning environment, otherness, boundary crossing, identity construction 
